\title{
Kazalo imena
}

\section{A}

Adam, Robert 133, 485

Afanasjev, S., fotograf 28

Abramić, Mihovil 33, 267, 440

Ajdačić, Dejan 167, 170

Akšamović, Antun 298

Alač, Jakov 396, 405

Alačević, obitelj 439

Albert-Jean 174

Alfieri, Vittorio 165

Alfirević, Frano 157, 165, 168, 171, 173 , $175,181,185,187,192,193$

Alfirević, Silvije 64

Alighieri, Dante 111, 160, 161, 167, 241, 356, 357

Almirante, Luigi 187

Amendola, Giovanni 331

Andersen, Hans Christian 356

Andonovska, Biljana 164, 168

Andrews, Philip 26

Andrić, Ivo 64, 161, 173, 175, 185, 186, 187, 192, 243

Andrić, Nikola 173, 181

Angjelinović, Budislav Grga 64

Anić, Nikola 332, 345

Antolović, Michael 268

Antonini, Ivan 332

Antonini, Otto 23

Antunac, Grga 138

Aragon, Louis 175, 177

Aralica, Stojan 80

Aralica, Višeslav 234, 244

Arduini, Luigi 349

Arendt, Hannah 235, 244

Ariosto, Ludovico 165

Armellini, Carlo 357

Armont, Paul 174

Arnold, Dana 136, 142

Artuković, Andrija 401, 403

Augustin Aurelije, sv. 63

Aymé, Marcel 175, 176

\section{B}

Babić, Ljubo s 78, 188

Badoglio, Pietro 359, 360, 361

Baglivi, Đuro 215

Bahr, Hermann 7, 8, 65, 68, 74

Baj, Rikard 197

Bajčeta, Vladan 19, 416, 455, 475

Bajohr, Franz 328, 346

Bakarić, Vladimir 331, 388-389, 397, 398, 411

Bakić, Dragan 312, 324

Balić-Nižić, Nedjeljka 10, 17, 168, 352, 361, 362

Baloković, Zlatko 39

Balzac, Honoré de 174, 175

Baljak, Dušan 432

Baljkas, Ivo 11, 61, 70-72, 74, 315

Banac, Ivo 233, 244, 252, 262, 315, 324

Banašević, Nikola 420, 422

Banović, Stipan 107, 109, 112, 116, 117

Banjanin, Jovan 312

Barac, Antun 188

Barač, Josip 197

Barać, Stanislava 15

Barante, Proper de 114

Baras, Frano 69,74

Barbieri, Frane 352

Barbusse, Henri 175

Barca, Calderón de la 67, 191

Bareris, Angelo 238

Barić, Daniel 492

Barić, Joško 168

Barić, Nikica 326, 345, 370, 371, 378, 394, 385, 396, 405, 410, 413, 487

Barrès, Maurice 238

Bartolucci, Athos 350

Bartulović, Niko 33, 64, 84, 99, 112, 157,

$158,160,162,168,187,201-202,204-$ 207, 209, 210, 266, 267, 281, 312, 333

Basić, Ivan 312, 324, 443, 456

Baschet, Jacques 175

Bastianini, Giuseppe 168, 336, 338, 340, $342,35,358$ 
Bastiansen, Marco 352, 362

Bataille, Henry 174

Batinica, Mile 431

Batistić, Ivo 197, 210

Batušić, Nikola 34, 42

Baudelaire, Charles 123, 17, 181

Bauerkämper, Arnd 394, 413

Bazin, Hervé 176

Beccadelli, Lodovico 165

Becuzzi, Emilio 332, 343

Bego, Krunoslav 292, 303

Bego, Marin 64, 189, 446, 488

Bego, Vladimir 32, 483

Begović, Božena 171, 173, 175, 181, 352

Begović, Milan 64, 67, 157, 161, 163, 165, $168,171,173,174,175,180,181,185$, 187-188, 190, 192, 193, 266, 299

Beker, Miroslav 230

Belan, Jerka 171, 173, 175, 181

Bell Villada, Gele H. 120, 129

Beller, Manfred 132, 142

Belli, Giuseppe Gioacchino 352

Benčić, Vladimir 28

Benda, Julien 15, 237, 244, 245-261, 262, 263, 486

Beneveni, Lorenzo 357

Benussi, Cristina 361, 362

Berelson, Bernard 64

Bergson, Henri 77, 84, 112

Berković, Josip 64, 310

Bersa, Josip 434, 445

Berus, Anka 71

Berus, Niko 417

Bervaldi, Josip 288, 289, 290

Besarević, liječnik 402

Bešević, Ivanka 425, 455

Bešker, Inoslav 10, 67, 74, 484

Bettiza, Vincenzo (Enzo) 353

Bezić, Ante 249

Bezić, Živan 285, 286

Bezić Božanić, Nevenka 35, 42, 187, 189, 193, 197, 201, 205, 210

Biankini, Juraj 23, 27, 218

Bibić, Petar 80

Bibó, István 236, 244

Bićanić, Rudolf 159, 169, 308

Bilinić, Pavao 88

Billetdoux, Raphaële 175
Billroth, Theodor 215, 216

Binički, Franjo 161

Blažević, Jakov 332

Boban, Rafael 387

Bobich, Luigi (Gica) 356, 360

Bobinac, Marijan 391

Bogdan, Ivo 373-374, 384

Bogdanović, Milan 220, 221-222, 223, 230

Bogner-Šaban, Antonija 67, 74, 198, 210

Boiardo, Matteo Maria 165

Boito, Arrigo 356

Bokanić, Trifun 318

Bonacci Čiko, Silvije 77, 80, 84-85, 91, 98, 99, 484

Bonald, Louis de 114

Bonavia, Aurelio 336

Bonifačić, Antun 179, 385

Bonifačić, Kvirin Klement 320, 350

Bontempelli, Massimo 161, 162

Borčić, Goran 20, 24, 25, 28, 31, 32, 39, 42

Borelli, Frano 439

Borelli, Manfred 215

Borko, Božidar 447-448

Borovčić Kurir, Đordano 332

Borović, Ante 21, 23, 24, 25-40, 41, 42, 309, 483, 484, 486

Borozan, Nebojša 382

Bosnar, Mario 179, 182

Bosnić, fotograf 40

Bošković, Ivan 169, 206, 210

Bošković, Ruđer 239

Botić, Luka 101, 207, 292, 339, 353, 369, 416, 417, 486

Botteri, Giuseppe 358

Bourdieu, Pierre 15, 171, 172-173, 180, 181, $182,245,247,248-250,251,252,255$, 256, 258, 260-261, 262

Bourget, Paul 173, 181

Boyd, Andrew 63, 74

Božić-Bužančić, Danica 285

Božović, Grigorije 269-271

Božović, Ratko 258

Brabant, Willy 329

Bracc, Robert 188

Bracciolini, Poggio 256

Brajer, K., novinar 70 
Brajević, Vinko, don 64, 66, 69, 81, 97, 99, 267, 272, 274, 317

Branđolica, Tomislav 10, 17, 146, 155, 295, 304, 487

Branica, Vinko 327, 346, 355, 363

Braudel, Fernand 300, 304

Briggs, Asa 300, 301, 304

Brix, Emil 300, 304, 394, 414

Brkić, Josip 320

Brkljačić, Maja 394, 414

Broz Tito, Josip 179, 234, 315, 331, 332, 354, 369, 384, 385, 386, 390, 420

Brueghel, Pieter st. 84

Brunelli, Vitaliano 440, 455, 488

Bruno, Giordano 358

Buber, Martin 391

Budak, Mile 165, 319

Bui, Boris 20

Bukarica, Miljenko 82, 99, 291

Bukovac, Vlaho 213, 299, 423

Bulat, Edo, ministar 310, 335, 370-371, 381, 384, 406

Bulat, Gajo 67, 197, 320

Bulatović, Miljenko 425, 455

Bulić, Ante 16

Bulić, Frane, don 32, 39, 86, 215, 266, 267, 283, 287-289, 290, 292, 294-296, 297, 302, 304, 318, 319, 486

Bulić, Mate 320

Bulimbašić, Sandi 10, 83, 99

Buljan, Ana 259, 263

Buljanović, Vicko 315

Buonarotti, Michelangelo 161, 165, 241

Burguière, André 283, 394

Burckhardt, Jacob 293

Butor, Michel 175

Buzzi, Paolo 356

Bužan, Joso 213

Byron, Gorge Gordon 216

\section{C}

Cacan, Fikret

Cacan, Fikret 182

Caccamo, Francesco 326, 345, 350, 351, 362

Calderón de la Barca, Pedro v. Barca

Campanella, Tommaso 416, 417, 418, 455
Camus (Kami), Albert 152

Canistraro, Philip V. 353

Capogrosso, obitelj 318

Cappi, Fericciol 353

Capriolo, Ettore 158

Car, Marko 268, 269, 276, 281, 486

Car Emin, Viktor 111

Carancini, Luigi 336

Carar Bataglia, Attilio 358

Cardarelli, Vincenzo 108, 161

Carducci, Giosuè 111, 147, 154, 160, 161, $162,165,356,416$

Carli, Mario 354

Casanova, Giacomo 175

Cavalcanti, Guido 353

Cesarec, August 256, 283, 302

Cettineo, Ante 12, 107, 112, 114, 116, 157, $159,160,162,168,171,173,175,181$, 451

Cettineo, K. , urednik 160

Charcot, Jean Martin 215

Chartier, Roger 250

Chevrel, Yves 171, 172, 181, 182

Chirico, Giorgio de 78

Churchill, Winston 352

Ciano, Galeazzo 234

Cilić, August 189

Clodfelter, Michael 326, 345

Colletta, Lisa 133, 142

Collini, Stefan 283-284, 304

Compagnon, Antoine 251, 262

Corazzini, Sergio 161, 165

Coronelli, Vicenzo Maria 438, 440, 444, 441, 488

Crisomali, Urban 64

Crivelli, Fabio Maria 356

Crnčić, Menci Klement 213

Crnica, fra Ante 422

Croce, Benedetto (Kroče, Benedeto) 11, 12, 13 , 77, 82, 98, 107-116, 117, 119, 121124, 127-128, 129, 131, 138-141, 142, 149, 154-155, 161-164, 167, 169, 189, 192, 193, 233-234, 236-237, 243, 246, 250, 331, 416-418, 429, 446-453, 454, 455, 457, 458, 485, 488, 489

Cronia, Arturo 161

Cuzzi, Ivo 310

Cvekan, Petar 259, 262 
Cvetišić, Vjekoslav 32

Cvetko, Dragutin 426, 455

Cvetković, Dragiša 234, 307, 308, 314, 322-323

Cvijović Javorina, Ivana $135,142,146,147$, $155,156,159,169,180,182,186,194$, 254, 263, 266, 268, 281, 284, 295, 298, $304,312,320,324,416,417,418,426$, $455,456,461,468,475,487,492$

Cvitković, Ivan 402

Cvitković, Maca 402

Cvitković, Viktor 401

Cvrlje, Zdenko 327, 345

Czerwinski, Maciej 234, 244

\section{Č}

Čale, Frano $185,186,188,193$

Čapo Žmegač, Jasna 136, 142

Čedomil, Jakša 161

Čerina, Ante 332,340

Čerina, Vladimir 157, 161, 165, 168, 266

Čičin Ša[j]in, Ćiro $12,69,81,82,84,85$, 99, 107, 111, 112, 116, 160, 451, 457

Čikoš Sesija, Bela 213

Čogelja, Jerolim 11, 61, 69, 70, 71-72, 74

Čović, Marko 165

Čulić, Jerko 39

Čulić, Paško 31-32

Čulić, Prosper 33

Čvrljak, Krešimir 135, 142

\section{Ć}

Ćelap, Đorđe 177, 179, 485

Ćipiko, Koriolan 197

Ćurčin, Milan 33, 234, 321-322

Ćurić, Juraj 66

Ćurin, Ivo 344, 345

Ćurin, Miroslav 326, 327, 334, 344, 345 , $346,347,382,390$

\section{D}

Dabac, Tošo 402

Dabit, Eugène 175

Dagelić, Ante 366
Dalmazzo, Renzo 351

Damiani, Enrico 160

Damjanović, Danilo 327,346

D'Annunzio, Gabriele 147, 160, 161, 165, $167,191,233,240,241,243,353,354$.

358, 416

Dante v. Alighieri, Dante

D’Anversa, Igino 352, 361

Darnton, Robert 250

Daudet, Alphonse 173,174

David, Filip 250, 263

Davidović, Ljuba 267

Davidson, Julije 25

De Amicis, Edmond 161, 166

Deanović, Mirko 161, 163, 434, 455

Dedijer, Vladimir 267

Dellale, Ante 111

Delalle, Ivo 111

Delorko, Olinko 112-113, 163, 165, 185, 186, 189, 192, 193, 417

Demeter, Dimitrije 217, 228

De Sanctis, Francesco 154, 161

De Vigny, Alfred 161

Descartes, René 147, 416-418, 455

Desnica, obitelj 20, 265, 278

Desnica, Boško (Barba) 5, 114, 117, 147, $154,155,163,278,319,417,422,422-$ 426, 428, 429-445, 450, 453, 454, 455

Desnica, Stojan 349

Desnica, Uroš (Ćaća) 14, 15, 20, 211, 214216, 229, 278, 312, 313, 324, 425, 488

Desnica, Vladan $8,10,12,13,14,15,18$, $19,63,67,69,77,86,97,98,99,100$, 107-108, 112, 114-115, 119-128, 129, 131142, 145-155, 157, 162-164, 166-167, 168, $171,173,176,177,179-181,182,185$, 186, 192, 193, 195-208, 209, 211-212, $214,229,246,256,279,280,312-313$, $319,415-453,454,455,456,458,459-$ 481, 485, 487, 488, 489

Desnica, Zorka 428

Desnica rođ. Carić, Ksenija 415, 425, 450

Dešković, Branislav 299

Dežman, Milivoj 234

Diehl, Charles 293

Dietrich, Wolfgang 431, 455

Dimitrijević, Mila 189

Dimović, Milan 201 
Dioklecijan 268-269, 272-275, 279, 290

Divoire, Fernand 174

Dizdar, Zdravko 354, 363, 408, 414

Dobrović, Petar 80

Doležel, Lubomir 472, 475

Deledda, Grazia 162

Dominis, Markantun de 239

Donadini, Ulderico 69, 161

Draganja, Vlaho 299

Dragman, Ervina 190

Dragojević, Nataša 182

Drinković, Mate 296

Dučić, Jovan 147

Dudovich, Marcello 87

Dugački, Vladimir 214

Du Gay, Paul 132, 142

Duhamel, Georges 175

Duišin, Ignacije 366, 382

Dujmić, Mirko 332

Dujmo, sv. 290

Dujšin, Dubravko 190

Dukić, Davor 135, 142

Dukić, Josip 290, 304

Dulibić, Frano 11, 78, 80, 88, 99, 100

Dumas, Alexandre 62

Duplančić, Arsen 31, 33, 42

Duplančić, Branko 332

Dupouy, Auguste 173

Durman, Milan 167

\section{$\oplus$}

Đilas, Milovan 332

Đorđević, Bojan 14, 188, 411, 485

Đorđević, Irinej 415, 426, 430, 454

Đujić, Momčilo 333, 341

Đurašković, Stevo 15, 235, 244, 252, 263 , 331, 346

Đurić, Dušan 266, 267, 271, 281

Đurić, Željko 148, 155

Đurić-Klajn, Stana 426, 455

\section{Dž}

Džugašvili Staljin, Josip Visarionović 179, 384

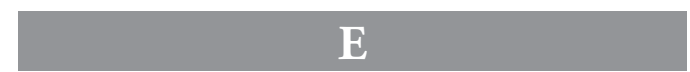

Eco, Umberto 62, 74, 361, 363

Ehrenburg, Ilja 384

Eichenberg, Ariane 393, 414

Einstein, Albert 102

Elias-Bursać, Ellen 186, 193

Engelbrecht, Martin $441,442,443,488$

Engelsfeld, Mladen 223, 230

Enzensberger, Hans Magnus 64

Epiktet 67

Eškinja, partizan 399

Eucken, Rudolf 195,210

\section{F}

Fallet, René 175

Fanti, likovni umjetnik 358

Febvre, Lucien 283

Felice, Renzo de 352, 363

Ferrero, Gina 236

Ferrero, Guglielmo 138, 162, 167, 233, 234, 236-237, 243

Ferrero, Leo 236

Ferrero, Nina 236

Ferruccio Tommaseo, Anton 359

Feyel, Gilles 62, 63, 74

Fijan, Andrija 189

Filipović, Rudolf 220, 230

Fiorino, Mario 356, 358

Fisković, Cvito 318-319, 451

Fischer, Torben 393, 414

Fiumi, L., urednik 160

Flaker, Aleksandar 178, 182

Flaker, Vida 159, 169

Flaubert, Gustave 123, 124, 174, 416

Fleck, Hans-Georg 235, 244, 331, 346

Flint, Robert 195, 210

Fogazzaro, Antonio 162

Fonseca Pimmentel, Eleonora de 356

Foretich, Bonaventura 336

Foretić, Vinko 197

Fortis, Albert 12, 134-135, 137, 140, 141, 142,440

Foscarini, Girolamo 444

Foscolo, Leonardo 437, 439, 444

Foscolo, Ugo 147, 154, 162, 165, 166 
Fotez, Marko 190, 196, 352

Foucault, Michel 261

France, Anatole 175

Frangeš Mihanović, Robert 213

Franičević, Marin $71,72,451,452,456$, 489

Franjo Asiški, sv. 147

Franz, Oskar 87

Franz, Otmar 326, 347

Friedman, Jonathan C. 328

Fritsch, Willy 39, 484

Frua, Dušan 332

Fržop, Krešimir 405

Fržop, Zvonimir 405, 406

Fuis, Franjo 40, 41

G

Gabričević, Branimir 192

Gaj, Ljudevit 312

Gale, Ivan 341

Galić, Ivan 21, 24, 25, 30, 31, 32, 34, 35, $36,42,79,80,357,483,484$

Galogaža, Stevan 188, 190

Gallone, Carmine 360

Galović, Fran 283, 302

Gamulin, Ćiro 319, 340, 341, 357

Gamulin, Grgo 97, 99

Gandhi, Mahatma 66

Gavella, Branko 188

Gavranić, Pavao 23, 483

Gecan, Vilko 79

Geiger, Vladimir 410, 414

Genasta, M., prevoditelj 161

Gentile, Emilio 238, 244

Gerbidon, Marcel 174

Gerić, Vladimir 220

Geršković, Leo 353

Gesemann, Gerhardt 419-420, 422

Ghéon, Henri 176

Gide, André 13, 171, 176-180, 181, 182, 234, 246, 250, 485

Girometta, Umberto 30

Giuliotti, Domenico 161

Giunio, Silvestar 64

Giunta, Francesco 336
Gizdić, Drago 350, 354, 358, 363, 381, 382, 390

Glavina, Ladislav 111

Gliubavaz, Simeone v. Ljubavac, Šimun

Globačnik, Matko 10

Glumac, Sergije 23

Goati, Vladimir 258

Goebbels, Joseph 366, 370

Gogh, Vincent van 84

Goldstein, Ivo 326, 346, 401, 410, 414, 492

Goldstein, Josip (Marko Giuseppe) 25, 28

Goldstein, Mary 21, 24, 42

Gorjanović-Kramberger, Dragutin 213

Gotovac, Jakov 423, 484

Govoni, Corrado 165

Gozzano, Aldo 161, 165

Gozzi, Carlo 319

Gramsci, Antonio 261

Grandits, Hannes 238, 244

Graovac, Igor 235, 244, 327, 331, 346

Grčić, Marko (Rako) 71

Grgić, Eduard 64

Grgić Maroević, Iva 13, 174, 186, 187, 191, 192, 193

Grgur Ninski (kip) 31-33, 86, 162, 266, $272,337,353,483$

Grimaldi, Giuseppe 337

Grizogono (Grisogno), Prvislav 272-274

Grković-Janović, Snježana 328, 346

Grol, Milan 196

Grossmann, liječnik 427

Gruber, Ivan 403

Guić, Antica 72

Guareschi, Giovanni 352

Gudehus, Christian 393, 394, 401, 414

Guéhenno, Jean 248, 251, 263

Gugenberger, Eduard 327, 346

Guilloux, Charles 175

Guinizelli, Guido 165

Gulbransson, Olaf 87

Gumhalter, Mira 312

Gundulić, Ivan 109, 112

Gunjača, Stjepan 294, 304, 318-319, 323

Guyard, Marius-François 171, 172, 181, 182

Gvozden, Vladimir 12,120 


\section{H}

Habermas, Jürgen 273

Hafner-Gjermanović, Ela 34

Hall, Stuart 132, 142

Haler, Albert 12, 107, 109-110, 112, 116, $117,163,164,169,451,489$

Hanisch, Ernst 300, 304

Harambašić, August 217, 220

Hartmann, Lud Moritz 294

Hatin, Eugène 62, 63, 74

Hatze, Josip 198, 299

Hebrang, Andrija 331

Heidegger, Martin 248

Heilbron, Johan 171, 173, 181, 182

Heinzel, Ret 294

Hektorović, Petar 291

Helm, Brigitte 39

Hergešić, Ivo 171, 172, 174, 181, 182, 228, 230, 248, 263

Hermand, Jost 370,378

Hitler, Adolf 267, 325, 327, 331, 369, 388, 395, 487

Hoffmann, Antonio 336, 340

Homer 110, 135, 215, 471

Höpken, Wolfgang 394, 414

Horacije 216

Horvat, Ivo 178, 181

Horvat, Josip 8, 62, 69, 74, 381

Horvat, Joža 154

Horvat, Romana 410, 414

Hržić, Vera 189

Hugo, Victor 173, 174

Huszár, Tibor 236, 234

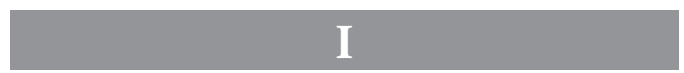

Ibler, Mladen 414

Igarashi, Hitoshi 158

Iljf, Ilja 66

Ilijić, Stjepko (Stjepan) 112, 157, 161, 162, 165, 166, 168, 185-186, 192, 193

Illich, Ivan 431, 455

Istrati, Panaït 175

Ivačić, Ante 162

Ivanić, Dušan $148,151,155,156$

Ivanišević, Drago 114,180
Ivanko, Juraj 86, 99

Iveković, Mladen 249, 256, 263

Iveković, Oton 213

Iveljić, Iskra 284, 304

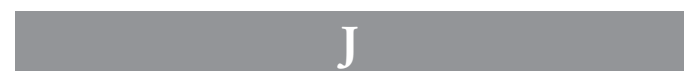

Jacquemard, Serge 175

Jacquette, Dale 139, 142

Jagatić, Nikola 404

Jagić, Vatroslav 215

Jakir, Aleksandar 16, 20, 146, 155, 159, 169, 298, 304, 307, 310, 314, 316, 321, 322, 324, 326, 328, 346

Jakopič, Rihard 29

Jakša, Vladimir 204, 205

Janeček, Gustav 213

Janić Capo, Vlado 332

Janićijević, Miloslav 249, 263

Janković Mitrović, rod 437

Janković, Branimir 15, 247, 261, 262, 263

Janković, Stojan 437, 438-439

Janović, Špiro 328, 330

Janović-Wagner, Lujza 328, 330

Jelaska, Petar 332

Jelaska, Vicko 64, 70, 315

Jelaska Marijan, Zdravka 21, 22, 33, 36, 42, 69, 74, 169, 174, 182, 266, 270, 281, 295, 299, 304, 307, 324

Jeličić, Živko 72

Jelić, Ivan 332, 346

Jelić, Luka, don 290

Jelić-Butić, Fikreta 308, 310, 315, 324, 333, 346, 394, 414

Jeremić, Jovan 207

Jergović, Miljenko 132, 140, 141, 142

Jerolim, sv. 239

Ježić, Slavko 174

Job, Ignjat $84,96,98,103,266,276,278$, 484, 485

Jonić, Vladimir 371

Jouhaud, Christian 62, 74

Jovanović, Ljubiša 190

Jovanović, Radul 258

Jozefović, Oskar 196

Jurašin, Miro 32, 38

Jureša, Ante 167 
Jurević, Ante 171, 173, 174, 181, 317

Jurić, Hrvoje 259, 261, 263

Jurišić, Šimun $71,72,74,168,169,189$,

193

Just-Verdus, Antonio 338, 350

Juvančić, kapetan 399, 405

\section{K}

Kalanj, Rade 247, 248, 251, 253, 258-259, 263

Kaliterna, Fabjan 31

Kaliterna, Paško 64, 310

Kálmán, Emmerich 34-35, 484

Kangrga, Milan 63, 74

Kant, Immanuel 125, 126

Karađorđević, obitelj 27

Karađorđević, Aleksandar 266, 272, 311, 454

Karađorđević, Andrej 29

Karađorđević, Marija 29

Karađorđević, Pavle 32, 271, 272

Karađorđević, Petar I. 23

Karađorđević, Petar II. 322

Karađorđević, Tomislav 29

Karaman, Josip 197, 299

Karaman, Ljubo 239, 288, 318, 319, 321, 323, 324, 486

Kardelj, Edvard 332, 385-386, 389

Karlovac, Mirko 371

Kasche, Siegfried 395

Kaštelan, Jure 157, 160

Katalinić, Blaženka 207

Katalinić, Jozo 366

Katalinić Jeretov, Rikard 111, 112, 162, 299

Katić, gđa 38

Katić, Ante 299, 332, 484

Katić, Jerolim 396

Katić, Lovro 318, 323

Katunarić, Ante 95

Katunarić, Dražen 182, 259, 263

Katunarić, Selma 300, 305

Katušić, Ivan 160, 360, 363

Kavanjin, Jerolim 101, 292

Kavurić, Lada 87,99

Kažotić (Casotti), Marko 162
Kečkemet, Duško 21, 22, 24, 25, 26, 28, $29,30,31,32,40,41,42,43,65,74,79$, $80,81,87,99,133,136,138,142,169$, 266, 171, 281, 299, 304, 317, 318, 324, 326, 346

Keitel, Wilhelm 366

Kerensky, Aleksandar 235

Khuen-Héderváry, Dragutin 287

Killinger, Charles L. 139, 142

Kinney, David 327,347

Kirigin, Josip 72

Kisić, Vinko 68, 69, 83, 99, 197, 215, 230, 267

Kisić Kolanović, Nada 298, 304, 326, 346, 356, 363

Kiš, Danilo 258, 263

Klaić, Mihovil 215

Klaić, Vjekoslav 294

Kleist, Heinrich von 352

Klement VIII., papa 357

Kletzin, Brigit 326, 346

Klimt, Gustav 87

Kljajić, Stipe 249, 256, 263

Kljaković, Jozo 78, 80, 86, 88, 98, 317, 321

Kolar, Slavko 319, 401

Kolešnik, Ljiljana 32, 43

Kolveshi, Željka 23, 43

Komenský (Comenius), Jan Amos 63

Končar, Rade 331, 332

Korać, Stanko 455

Korb, Alexander 328, 346

Korolija, Dušan 523, 424

Korolija, Mirko 121, 10, 202, 203-208, 209, 416, 423, 431, 434

Kortšek, Josip 11, 61, 69, 70, 71-72, 74

Kosanović, Dejan 39, 43

Kostić, Dragutin 197, 208, 209

Kostović, Jakov 111

Koščević, Želimir 24, 43

Košutić-Brozović, Nevenka 182

Kotur, Goran 327

Kovač, Tatjana 366, 380, 390

Kovač, Zvonko 284, 301

Kovačec, August 196

Kovačević Lala, Mirko 332, 334

Kovačić, Branko 67

Kovačić, Davor 402, 403, 414

Kovačić, Ivan 295, 298-299, 304 
Kovačić, Ivan Goran 163, 248

Kovačić, Joško 215

Kožičić, Šimun 421, 488

Kragić, Bruno 11

Kralj, Bogdan 291

Kraljeva, Božena 190

Kraljević, Marko, junak 420

Kraljević, Miroslav 443

Kranjčević, Silvije Strahimir 111, 161

Kraš, Josip 332

Kravar, Miroslav 319

Kravar, Zoran 78, 99, 230

Krekich, Natale 357

Krišković, Vinko 220, 221-225, 230

Krizman, Bogdan 326, 346, 405, 414

Križanić, Juraj 241

Križanić, Pjer 278, 279

Krleža, Miroslav 115, 178, 180, 243, 252, 255-257, 260, 263, 283, 302, 448

Krnjević, Juraj 316

Krolo, Petar 20, 365

Krstanović, Oton 267

Krstelj, Ivo 201

Krstić, Miloš 327, 347

Krstulović, Andrija 138, 140

Krstulović, Vicko 315, 332, 346, 381, 390

Kršnjavi, Izidor 312

Kruševac, Čeda 267

Kučera, Elza 318, 324

Kudrjavcev, Anatolij 21, 43, 65, 66, 68, 74, 101, 102, 105, 169, 203, 205, 210

Kuhačević, Mateša 161

Kuić, Ivanka 20, 66, 74, 267, 281, 350, $363,372,378$

Kuić, Jure 34, 35, 42, 43

Kukuljević Sakcinski, Ivan 293, 425

Kulčar Prut, Antonija 24

Kulušić, Joško 71

Kuljiš, Tomislav 34, 35, 42, 43

Kumičić, Eugen 66, 287, 293, 303

Kurelac, Miroslav 440, 456

Kušan, Jakša 160, 235

Kuzmić, Marin 68, 75, 168, 286, 326, 327, 345, 346, 353, 360, 362, 378, 486

Kvaternik, Eugen Dido 402

Kvaternik, Eugen 292, 310, 312, 402, 403
Kvesić, Sibe $315,322,324,326,346,382$, 383, 390

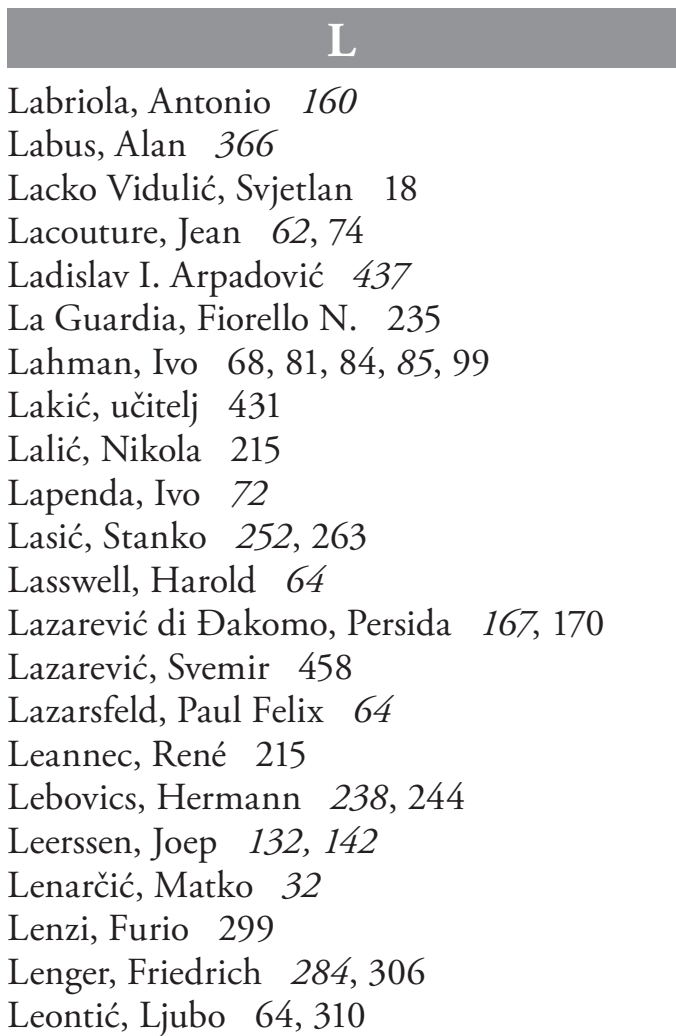

Leopardi, Giacomo 147, 152, 154, 161, 163, $164,165,167,353,416,485$

Lepenies, Wolf 247

Le Rider, Jacques 492

Leskošek, Franc 332

Levi, Jirži 230

Lewy Goldstein, Violeta 21, 24, 42

Livolsi, Marino 62, 74

Lombroso, Cesare 236

Loose, Ingo 328, 346

Lorenz, Matthias N. 393, 414

Lorković, ministar 406

Loti, Pierre 175

Lovrić, Božo 66

Lozica, Ivo 138

Lozovina, Vinko 66, 111, 157, 161-162

Luburić, službenik 402

Lucić, Hanibal 291

Lucio, Giovanni v. Lučić, Ivan

Lučić Ivan 437, 438, 490, 444, 445 
Lučić Lavčević, Ivan 353

Luger, Herkulan 64

Lukas, Filip 162, 234, 241

Luxardo, Emanuel 215

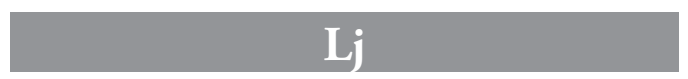

Ljubavac, Šimun 437, 438, 439, 440-445, 453, 488

Ljubičić, Saša 295, 304

Ljubić, Pero 112

Ljubić, Šime 440

Ljubljanović, Srećko 71,74

\section{M}

Macan, Trpimir 380, 390

Machiavelli, Niccolò 111, 161, 356

Machiedo, Jerko 215

Machiedo, Tereza 215, 216

Machiedo Mladinić, Norka 21, 23, 37, 39, 40,43

Maček, Vladko 234, 307, 308, 310-311, 313, 315, 317, 320, 322-323, 343, 388, 389, 429, 430, 487

Maeterlinck, Maurice 174

Magaš Bilandžić, Lovorka 10, 24, 43

Maitland, Frederic William 284, 304

Majstorović, Božo 21, 43

Maldini, R. B., prevoditelj 173

Maleković, Vladimir 24, 42

Mallardo, Giuseppe 336, 353

Mallarmé, Stéphane 124, 174

Manger, Dušan 297, 450, 489

Mann, Thomas 111, 117

Mannheim, Karl 261

Maras, Mate 220, 221,222,225,230

Marasović, Ante 353

Marchi-Markić, Ivan Petar 285

Marcocchia, Giacomo 160, 169

Maretić, Tomislav 312

Margetić, Viktor 370

Marić, Ivan 70, 315

Marinetti, Filippo Tommaso 161, 162, 165 , 354

Marinković, Dušan $146,155,176,179,162$, $416,417,418,423,425,434,455,456$
Maritain, Jacques 234, 237

Marjanović, Milan 32, 299

Markišić, Aleksa 267

Markotić, Ivan 332

Markotić, Josip Jozo 332

Marković, Franjo 293

Marković, Mihajlo 201

Marković, Slavica 87,99

Markovina, Jelena 33, 43, 335, 338, 346

Maroević, Tonko 176, 465, 475

Marović, Duško 37, 43

Martić, Grga 272

Martinis-Marchi, obitelj 285

Martinović, M., svjedok 409

Marulić, Marko 30, 31, 42, 101-102, 241, 291, 292, 483

Marušić, Ante 352

Marušić, F. D. 309

Masaryk (Masarik), Tomás Garrigue 248

Masovčić, Ante 443, 444

Masson, Jean-Yves 171, 172, 181, 182

Matačić, V., novinar 70

Matavulj, Simo 66, 147, 424, 425

Matić, Lazar 439, 431, 432

Matijević, A., pripadnik stranke 310

Matković, Marin 332

Matković, Stjepan 312, 324

Matoš, Antun Gustav 78, 99, 83, 288, 302

Matošić, Joe 69, 291

Matošić, Vlado 314

Matović, Vesna 164, 168

Matsuo, K., urednik 160

Matthäus, Jürgen 286m 328, 346

Maupassant, Guy de 352

Maurano, Silvio 336

Mauriac, François 176, 237

Maurović, Andrija 160,173

Maurras, François 238, 239

Maykowky (Majkovski), Ipolit Danilovič 80,358

Mazower, Mark 326, 346

Medici, Lorenzo de 165

Medović, Celestin 213, 289, 297, 299

Mekinić, Ante 68,75

Meneghello Dinčić, Virgil 77, 87, 98, 299

Meneghello Rodić, Mate 77, 86, 87, 93, 98, 357,484 
Menini, Giulio 160, 169

Meštrović, Ivan 12, 30, 31-44, 78, 80, 86, 88, 98, 131, 132, 136-140, 141, 142, 197, 213, 246, 266, 270, 271, 272, 274, 299, 317-318, 321, 322, 323, 324

Michelangelo v. Buonarotti, Michelangelo Mignet, François 293

Mihailović, Draža 333, 354, 369, 410

Mihaljević, Nikica 260, 263

Mihaljević, Vicko 66, 67, 243

Mihičić, Milica 190

Mihić, kotorski predstojnik 408

Mihovilović, Ive 71,288

Mihovilović-Šusta, Olga 297

Mileta, Jerolim 397

Miletić, Branko 404

Miličić, Josip Sibe 157, 165-166, 168, 169

Milijanović, S., novinar 202

Milović, Jevto M. $146,416,423,424,425$, 456

Milutinović, Ivan 332

Miljan, Goran 389

Miljković, Branislav 109

Miljuši, pripadnik političke stranke 432

Miović, Vesna 287, 304

Mirković, Ivan 80, 84, 173

Mirković, Vojko $68,70,71,72,75$

Mistral, Frédéric 233, 238, 239, 243

Miše, Jerolim 80, 81, 90, 98, 112, 484

Mitropan, Petar 421

Mitrovići Jankovići v. Janković Mitrović

Molière (Jean Baptiste Poquelin) 178, 228

Moller, Sabine 392i, 414

Montale, Eugenio 165

Monti, Vincenzo 165

Monzali, Luciano 326, 336, 340, 345, 346, $350,351,353,354,357,362,363$

Morđin Crni, Ivan 387

Morgagni, Mario 359

Morović, Hrvoje 169, 297, 304

Morpurgo, obitelj/knjižara 159, 160, 166, 343, 357, 485

Morpurgo, Luciano 160, 169

Morpurgo, Vito 159

Mosinger, Franjo 24

Mrkonjić, Dinko 28

Mrkonjić, Zvonimir 176

Müller, Rolf-Dieter 325, 346
Munzani, Petar Dujam 397, 398, 411

Muratori, Antonio 293

Mussolini, Benito $160,234,325,326,331$, 336-339, 345, 350, 352, 353, 354-355, $358,359,360,361,375,388,395,487$

Mussolini, Bruno 338, 352

Mužinić, Zdravko $159,161,162,169,170$

\section{N}

Nagy, Ferenc 235

Nagy, Josip 308

Naisbitt, John 64

Najbar-Agičić, Magdalena 180, 182, 381

Nardelli, Bruno 350

Nazor, Josip $165,174,180$

Nazor, Vladimir 157, 158, 159, 168, 169, 171, 173, 174, 181, 359

Nedeljković, Iv 258

Nedić, Marko 164, 168

Nedić, Milan 329

Negri, Ada $\quad$ 161, 165

Nemec, Krešimir 284, 304

Nerici, Bartoloméo 133, 485

Nevistić, Franjo 298, 304

Niccodemi, Dario 187, 188

Nietzsche, Friedrich 77, 84

Nightingale, Florence 328

Nikolić, T., fotoamater 32

Nikolić, Vilko 298, 304

Nikolić, Vinko 235, 319

Ninski, Grgur v. Grgur Ninski

Nizan, Paul 175, 177

Nizeteo, Anton 157, 163, 164, 165, 168, 185-186, 188, 192, 193

Nodilo, Natko 135-136, 291, 293, 294

Novak, Božidar (Božo) 69, 75, 366, 378, 380, 381, 382, 390

Novak, Grgo 159, 160, 169

Novak, Slobodan Posperov 236, 244

Novak, Viktor 294, 366, 367, 371, 373

Novaković, Fran 207

Novaro, Angiolo Silvio 165

Novović, Mirko 327, 346

Noworyta, Stanislav 24

Nučič, Hinko 189, 190

Nušić, Branislav 198-202, 205, 209 


\section{O}

Obradović, Dositej 126, 422, 434

Odić, Slavko 371

Ogrizović, Milan 199

Olschowsky, Burkhart 328, 346

Olujić, Grozdana 425, 456

Omero, Violeta 21, 24-25, 27, 42

Orešković, Marko 332, 333

Oriani, Alfredo 352

Orsini, Gian Napoleone Giordano 138, 142

Ortega y Gasset, José 233, 234, 237, 240 241, 243, 244, 252-253, 256-257

Osmanagić, Munib 312

Ostrovski, Nikolaj 166

Overy, Richard 326, 346

Ozretić, Vjekoslav 332

\section{P}

Polić, Krešimir P. 422

Ponte, Valerio da 441

Pulić, Mate 439

Pailleron, Édouard 174

Palazzeschi, Aldo 108, 161, 165

Pancrazzi, Pietro 161

Pandurović, Sima 199-201, 208, 209, 485

Panozzo, Giovanni 62, 74

Pap Šilja, Pavle, komunist 332

Papini, Giovanni 108, 111, 161-162, 285

Parać, Ivo 112, 165, 353, 416, 417

Parać, Vjekoslav 77, 78, 80, 85-86, 92, 98, 100, 112, 317, 321, 484

Parčina, N., profesor 365

Parini, Giuseppe 165

Parmenid 152

Pascal, Engel 253, 263

Pascoli, Giovanni 160, 161, 165, 167, 353

Pasini, gosp. 428

Pasquinelli, Maria 355

Pasteur, Louis 215, 216

Paulhan, Jean 176

Pavelić, Ante 329, 369-371, 363, 386, 390, 395, 401

Pavić, Josip 34, 189

Pavletić, Vlatko 132, 142, 162, 169, 423, 424,456
Pavličić, Pavao 230

Pavlović, Cvijeta 174, 182

Pavlović, Marin 357

Pecenko, L., pisac 352

Pederin, Rudolf 309, 310

Pelaić, Marin 326, 334, 335,336, 346

Pellico, Silvio 161

Penić, Dujam 77, 96, 97, 98, 112, 484

Perica, Vjekoslav 68

Peričić, Božo 14, 211-229, 230, 427, 428, 432, 485

Peričić, Josip 212, 215, 216

Peričić, Helena 14, 211, 228, 230

Peričić, Šime $214,215,217,231$

Perković, Mirko 228, 231, 448

Pernar, fotoamater 40

Perović, Slavko 213, 231

Peršić, Ivan 288, 303

Petitjean de la Rosière, Frédéric 175

Petitjean de la Rosière, Marie 175

Petković, Stevan 327, 346

Petrarca, Francesco 165

Petrač, Božidar 285, 288, 305

Petravić, Antun 111, 161, 165, 169, 449

Petricic, Antonio 358

Petrić, Franjo 111

Petrić, Josip (Jozo) 332

Petrić, Hrvoje 492

Petrinović, obitelj 276

Petrinović, Mate 276

Petrov, Jevgenij 66

Petrović, Leo 298, 305

Petrović Njegoš, Petar II. 137, 469

Picasso, Pablo 78, 86

Pichois, Claude 171, 172, 181, 182

Pičman, Josip 273, 274

Pietromardi, Luca 352

Pinčić, Petar 399, 410

Pinkowa (Pinkova), Tereza 215

Piper, Ernst 327, 346

Piplović, Stanko $146,155,285,320,324$

Pirandello, Luigi $13-14,114,161,162,185-$ 192, 193, 485

Pistoia, Cino da 165

Pitigrilli 417 v. Segre, Dino

Plančić, Juraj 78, 88

Plečnik, Jože 318 
Pokorni, Dragutin 198

Poliziano, Angelo 165

Polonijo, Stanislav 385

Popadić, Momčilo 86, 99

Poparić, Bare 285, 286, 287-288, 289, 290, 292

Popović, Ante 332

Popović, Bogdan 274

Popović, Branko 267, 274, 278

Popović, Olivera 166

Popović, Pavle 80

Popović, Tanja 467

Popović, Vladimir (Vlado) 331

Porter, Roy 301, 305

Portmann, Michael 410, 414

Posavac, Zlatko 82, 99

Požar, Petar 70, 75, 266, 281

Prančević, Dalibor 31, 32, 43

Prelog, Petar 32, 43

Preradović, Petar 109, 110, 292

Prezzolini, Giuseppe 108

Pribićević, Svetozar 204, 206, 207

Prijatelj, Kruno 299, 305

Prlenda, Sandra 394, 414

Prodan, Ivo 287, 292

Proust (Prust), Marcel 152

Prudhomme, Sully 174

Puccini, Giacomo 352

\section{R}

Rabadan, Vojmil $114,165,171,173,175-$ 176, 181, 185, 186, 190, 192, 193, 320

Racine, Jean 178

Račić, dr Jakša 37, 64, 215

Rački, Franjo 312

Rački, Mirko 78, 299

Radetić, Josip 332

Radica, Bogdan 12, 15, 67, 75, 107, 108, 111, 112, 116, 117, 145, 146, 154, 157, $161,164,167,168,169,171,173,175$, $177,181,182,185,186,187,190-191$, 192, 194, 233-243, 244, 245-246, 247 , $249,250,251,252,255,280,262,263$, 288, 291, 298, 305, 331, 347, 418, 451. 456, 457-458, 485, 486, 489

Radica, Branko (Branislav) 239

Radica, Zlatka 352
Radić, Stjepan 67-68, 294, 310, 312

Radja, Mihovil 37, 43

Radojčić, Svetozar 469, 475

Radovanović, M., autor 276

Radulović, Jovan $148,151,155,156,462$, 475

Raffael Santi 241

Raić, Ivo 34, 189, 198, 201

Rakovac, Ladislav 213

Rambaud, Alfred Nicolas 293

Ramov rođ. Urschitz, Marija 397, 410

Ramov, Sonja 397, 411

Ramov, Viktor 18, 391-412, 413, 487

Rancière, Jacques 128, 129

Randi, Oscar, funkcionar 352

Randić, Ivan 353, 360

Ranković, Aleksandar 332

Ravlić, Jakša 157, 160, 168, 299, 305

Reberski, Ivanka 84, 99

Regan, Krešimir 311, 314, 315, 319, 324

Reichherzer, Frank 102

Reić, Lovre 327

Reisch, Emil 286

Renan, Ernest 216

Renaudot, Théophraste 62-53, 64

Rendić, Ivan 161, 299

Rendulic, Lothar 371

Repak, Salko 207

Ribar, Ivo Lolo 332

Rimac, Marko 312, 324, 456

Rimini, Francesca da 147

Rinuccini, Ottavio 165

Rismondo ml., Vladimir 12, 151

Rismondo, Vladimir $12,13,14,81,85$, 99, 107, 112, 114, 116, 131, 146, 150-151, $154,155,157,163,168,185,186,192$, 193, 184, 319, 451

Riškov, Viktor 189, 221

Rittig, Svetozar 238, 397, 399

Robić, Dunja 174

Roca, Stjepan 271

Roger, Noëlle 175

Rogulic, likovni umjetnik 358

Rogulj, Dragutin 332

Roić, Sanja 10, 13, 17, 147-148, 155, 156, 163, 167, 170, 180, 182, 186, 194

Roksandić, Drago 15, 18, 19, 37, 43, 86, $107,135,142,146,147,149,155,156$, 
$159,163,169,170,180,182,186,194$ $254,256,263,266,268,281,284,295$, $298,304,312,320,324,404,416,417$ $418,423,425,426,430,443,455,456$, 461, 465, 468, 475, 492

Rolland, Romain 108, 177

Romac, Stipe 332

Romano, Sergio 354

Rosandić, Tomo 138, 197, 266, 267, 274277, 280, 299, 486

Rosenberg, Alfred 327

Roosevelt, Franklin Delano 214, 352

Rossi, Pietro 352

Rosso di San Secondo, Pier Maria 352

Rousseau, André-Michel 171, 172, 181

Rubić, Ivo 31

Rudolf, Davorin 71

Rukavina, Ivan 331

Ruljančić, Jure 26, 483

Ruljančić, Petar 21, 24-26, 31, 42

Ruljančić, Zlata 25

Rushdi, Salman 158

Rušinović, Nikola 288, 305

Ružička-Strozzi, Marija 189

\section{S}

Saba, Umberto 165

Sabalich, Giuseppe 444

Sabić, Marin 112

Sagan, Françoise 175

Said, Edward W. 133, 143, 261

Saint-Exupéry, Antoine de 175

Salewski, Michael 326, 347

Salvemini, Gaetano 162

Sand, George 216

Santini, Alfred 332

Santo, Imre V. 87

Sapiro, Gisèle 171, 173, 181, 182

Sapunar, Ante 201, 210, 357, 363

Sapunar, Dora 201, 210, 357, 363

Sarkotić, Stjepan 312

Sartre, Jean-Paul 174, 175, 248, 261

Sasso, Antun 166

Savić, Milorad 437, 455

Savin, Drago 352

Savo, Giovanni 334, 336, 340, 486
Schiele, Egon 87

Schiller, Friedrich $12,120,160$

Scipion Afrički 356, 358

Schiessel (Šisel), Otmar 472

Schlegel, August Wilchelm 220

Schlegel, Toni 69

Schneider, Robert 289

Schönfeld, Enrico 443, 444

Schopenhauer, Arthur 139

Scurto, Ignazio 356

Segre, Dino 417 v. Pitigrilli

Sekulić, Isidora 267, 276

Selem, Stefan 176,350

Selmani, Nikša 287, 304

Selnik, Marina 411

Senjanović, Petar 64, 273, 274, 484

Serao, Matilda 161

Sertić, Tomislav 310

Sesar, Dubravka 211, 230

Sforza, Carlo 458

Shakespeare, William 67, 188, 211, 216225, 228, 229, 230, 485

Shaw, John Bernard 39

Sica, Vittorio de 360

Siebmacher, Johann 444

Silone, Ignazio $147,148,167,180$

Silva, Pietro 147, 416

Simić, Vladimir 450

Simone, Giovanni 147

Sindičić Sabljo, Mirna 132, 143

Singer, Lily 87

Sinko, Matija 442, 456

Sinobad, Stevan 21, 24, 25, 27, 28, 29, 32, $33,36,40,42,483$

Sirovica, Stjepan 213, 214, 215, 231

Skok, Petar 160

Skopljanac, Tadija 332

Slade, Iris $21,43,84,88,100$

Sladović, Ivana 409

Slijepčević, Pero 421

Smajić, Petar 77, 88, 96, 97, 98, 484

Smerdel, Ton 111, 161

Smodlaka, Josip $64,65,68,160,271,273$, 274-275, 343, 386

Smoje, Miljenko $67,71,72,75,243,299$, 300, 305

Snowman, Daniel 300, 301, 304 
Sobolevski, Mihael 408, 414

Soffici, Ardengo 161, 165

Soglian (Šoljan), Giovanni 354, 355

Solar, Milivoj 163

Soldati, Mario 352

Solitro, Giulio 66

Sorani, Settimio 357, 363

Spalatin, Stjepan 310

Spasojević, Zdravko 186

Spengler, Oskar 238

Spigo, Umbertol 343

Srhoj, Vinko 86, 100, 137, 143

Sroka, Otto 366, 374-375

Stajić, Aleksandra 466

Stalio, Ivo 36

Stalio, Stefanija 36

Staljin v. Džugašvili Staljin

Stamać, Ante 187, 194

Stampa, Gaspara 165

Stančić, Nikša $134,136,143$

Stanojević, Branko 161

Stanojević, Juraj 344

Starčević, Ante 292-293, 296, 310

Starčević, Maja 228, 231

Starčić, Viktor 207

Starova, Luan 258, 263

Stecchetti, Lorenzo 165

Steinbeiss, Otto von 215, 216

Steinhoff, Hans 39

Stendhal, Marie Henri Beyle 174, 175

Stepinac, Alojzije 266, 298, 397

Stipetić, Zorica 249, 256, 263

Stipčević-Despotović, Anđelka 312

Stjepan II. Trpimirović 437

Stojnić, Mila 231

Stojadinović, Milan 314, 430

Stojanac, Šimun 332

Stojanović, Sreten 278

Stopar, Vladimir (Vlado) 380, 390

Strossmayer, Josip Juraj 215, 312

Strozzi, Tito 190

Studin, Marin 138, 358

Stühler, Dragutin Karlo 21, 24-26, 28, 3032, 38, 41, 42, 483

Stuparić, Darko 394, 414

Styx, Paul 87

Subotić, Dragomir 40, 404
Subotić, Nedjeljko 83, 100

Subotić, Vojislav 215

Sue, Eugène 176

Sundhausen, Holm 394, 414

Supek, Rudi 179

Suppan, Arnold 394, 414

Supervielle, Jules 173, 175

Suppé, Franz (Francesco) von 357

Svevo, Italo 164,424

Szücs, Jenö 236, 244

Swift, Johnatan 61

\section{Š}

Šalov, Mate 327,347

Šamšalović, Gustav 160

Šeatović-Dimitrijević, Svetlana 12

Šegina, Duško 37

Šegota, Pero 85, 100

Šegvić, Kerubin/Cherubin (Matej Marin) $16,64,65,68,110,112,117,157,158$, $160,165,168,170,246,283-302,303$, 305, 486

Šegvić, Neven 72

Šenoa, August 67

Šeparović, Ana 11, 78, 88, 100

Šeparović Palada, Maja 32, 43

Šepić, Ante 417

Šesnić, Jelena 234, 244

Šestan, Mijat 399

Šimetin Šegvić, Filip 16, 486

Šimetin Šegvić, Nikolina 16

Šimić, Milan 28

Šimundža, Drago 182

Šimunović, Dinko 160

Šišić, Ferdo 291, 294

Šitin, Tonći 291, 294

Škurla Iliijić, Verka 112, 157, 162, 168, 171, 173, 175, 181

Šoštarić, Sanja 13, 253

Šporer, Juraj 217, 228

Šram, Ljerka 189

Šubašić, Ivan, ban $311,319,320$

Šufflay, Milan 309, 311

Švab, Mladen 70,75 


Tacconi, Antonio $334,350,353,358$
Tadić, Jorjo 160
Tafra, Alen 134,143
Tagore, Rabindranath 110
Taine, Hypolite 110
Talpo, Oddone $350,358,363,395$
Tartaglia, Ivo 23, 33, 64, 65, 83, 159, 160,
$\quad$ 197-199, 201-202, 205, 208, 209, 295,
317, 483
Tartaglia, Marino $83,98,100,317$
Tartaglia, Oskar 159,170
Tasso, Torquato 165
Taylor, Karin 238,244
Tecilazić, Vinko $171,173,175,181$
Teich, Mikulás 301,305
Tenfelde, Klaus 284,304
Thomas, Édith 175
Tieck, August 220

Tijardović, Ivo 23, 34-35, 77, 87, 98, 100, $188,189,190,196,320,321,323,484$

Tilgher, Adriano 162, 189, 191, 192, 194

Tit Livije 356

Tito v. Broz Tito, Josip

Todorović, Tićo 369

Tolić, likovni umjetnik 358

Tolić, Milan 77, 80, 86, 93, 98, 484

Toller, Ernst 110

Tolstoj, Aleksej 166

Toma Arhiđakon (Arciđakon) 61, 131, 142, 292, 297, 356

Tomasevich, Jozo 331, 333, 347, 395, 414

Tomašić, Dinko 420

Tomerlin, Slavko 80

Tomić, gruntovčar 402

Tomić, „koljač“ 399

Tomić, Ante 61

Tomić Ferić, Ivana 249, 305

Tommaseo, Ivan 359

Tommaseo, Niccolò (Nikola) 135, 239-240, 351, 359

Tommaseo (Tomazeo), Radovan 23, 77, 87, 98, 201, 483

Tommaseo, Ruggero 359, 361, 363

Tončić, Kamilo 30, 31, 299

Tonković, Marija 24, 25, 43

Torbarina, Josip $164,220,225,230$
Torcoletti, Luigi 421, 422, 488

Torkar, Ante 332

Tortić, Janko 402

Trajan 290

Trenk, Franjo 441, 443

Trepše, Marijan 79

Tresić Pavičić, Ante 111, 162, 165, 270, 288, 291, 294, 299

Trgo, Fabijan 327, 334, 347

Trnokopović Stojan, major 32

Trumbić, Ante 64, 65, 68, 215-216, 286, 294-295, 311, 385, 486

Tschuggnall, Karoline 392, 414

Tudor, Gordana 327

Tuđman, Franjo 260

Tveit, Eva-Marie 143

\section{U}

Ujević, Augustin (Tin) 7, 8, 11, 12, 64, 66, 80-82, 100-105, 107, 110-111, 116, 117, 157,159, , $160,161,162,168,171,173$, $174,180,181,182,185,186,187,192$, 194, 243, 246, 266, 268, 269, 485

Ujević, Mate 319

Unamuno, Miguel de 111, 175, 233, 239, 243, 252, 253

Ungaretti, Giuseppe 165

Urbani, Umberto 161, 420, 457

Urlić, Davor 332

Uršić, Adolf 403

Urukalo, Sergije 64, 341, 430, 431

Uvodić, Angelo 23, 31, 66, 77, 80, 88, 94, 98, 318, 357, 484

Uvodić, Marko 66, 159, 170, 243, 298

Uzelac, Milivoj 79

Užarević, Josip 158, 170

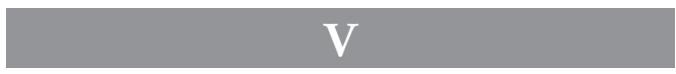

Vailland, Roger 175

Valdec, Rudolf 213

Valden, Jurij 166

Valerio, Théodore 137, 485

Valéry, Paul 152, 154

Valli, Alida 360

Van der Wijk, Nikolaus 419-420, 488 
Van Tieghem, Paul 171-172, 181, 182

Vaško, Tibor 405

Vavra, Nina 34, 188, 189

Vecchi, Gian Guido 62

Večerina, Duško 338, 347

Vekarić, Živko 162

Velikanović, Iso 61

Velzek, Ante 458

Venturi, Lionello 116, 117, 147

Verdi, Giuseppe 352

Vergani, Vera 187

Vergilije, Publije 216

Verhaeren, Émile 174

Verlaine, Paul 110

Vico, Gianbattista 148

Vidjak, Vjekoslav 327

Vidovich, Mario de 356, 363

Vidović, Emanuel 25, 66, 77, 78, 80, 83$84,89,98,112,140,141,197,213,299$, $317,321,323,484$

Vidović, Veljko 25, 40

Vidović Bolt, Ivana 211, 230

Villon, François 176

Vilović, Đuro 64, 333

Vinaver, Stanislav 278

Vinci, Leonardo da 356

Vincio, Vincenzo 337

Visoina, Livia 358

Višić, Vlasta 327

Višnjić, Čedomir 416, 456, 457

Vitezica, Vinko 12, 107, 111, 116, 163, 164, 169,447

Vojnović, Ivo 35, 66, 119, 188

Vojvodić, Jasmina 158,170

Volkelt, Johannes 109

Vrandečić, Josip 21, 43

Vrcan, File 64

Vrdoljak, S., novinar 38

Vučetić, Šime 451, 489

Vujadinović, Dragica 258

Vujošević, Jovan 166

Vukelić, Zvonimir 296

Vukićević, Dragana 151, 156

Vukonić, Boris 39, 43

Vukušić, Stjepan 361, 366, 371, 382
Vušković, Boris 326, 346

Vyslonzil, Elisabeth 394, 414

\section{W}

Waldecker, Burkhart 370

Walton, John K. 238, 244

Weichs, Maximilian von 371

Weixler, Viktor 87

Welzer, Harald 392, 393, 394, 414

Wilde, Oscar 123

Winton, Robert de 355

Wittman, Robert K. 327, 347

Wolfe, Pierre 174

\section{Z}

Zeiss, H., likovni umjetnik 318

Zajc, Ivan pl. 35

Zanella, Pero 449

Zaninović, Vice 319

Zečević, Ana M. 416, 457

Zelić, Ante 332

Zelić, Pavle 430

Zenon 152

Zerbino, Paolo 336, 337, 339, 340, 341, 353, 356

Zervas, Theodore G. 136, 143

Zibina-Jakaša, Meri 356

Zidić, Igor 83, 86, 100, 485

Zima, Zdravko 143

Zola, Émile 174, 175

Zoričić, Antun 220

Zrinski, Petar 293

Zuppa, Antun 72, 80, 84, 112, 318, 484

Zuppa, Vjeran 254, 263

Zwitter, Fran 248

\section{$\check{\mathrm{Z}}$}

Žanko, Miloš 382, 389

Žerjavić, Vladimir 326, 347

Žeželj, Mirko 159, 170

Žižić, Nela 25, 43

Žuljan, Marijan 327 